RESEARCH ARTICLE

\title{
Kinetics and characteristics of replication-competent revertants derived from self-inactivating foamy virus vectors
}

\author{
P Bastone and M Löchelt \\ Abt. Genomveränderung und Carcinogenese, Forschungsschwerpunkt Infektion und Krebs, Deutsches Krebsforschungszentrum, \\ Heidelberg, Germany
}

\begin{abstract}
In this study, self-inactivating (SIN) retroviral vectors based on feline foamy virus (FFV) were constructed and analysed. The FFV SIN vectors were devoid of the core FFV long terminal repeat promoter plus upstream sequences but contained all structural and regulatory genes. This design allowed sensitive detection of replication-competent revertants (RCRs). The FFV SIN vectors efficiently transduced the green fluorescence protein into recipient cells. However, RCRs appeared after serial passages of transduced cells. In all RCR clones analysed, parts of the heterologous cytomegalovirus immediate early promoter, originally driving expression of the FFV vector genome, were taken up to restore the deleted SIN promoter function required for replication
\end{abstract}

competence. The RCRs were strongly reduced in replication capacity compared with the parental replication-competent vectors containing the FFV promoter. In all RCR genomes analysed, the uptake of the heterologous promoter was accompanied by deletion of almost the complete marker gene. Although the RCRs described in this study may not have the capacity to spread in humans and animals, they may pose a theoretical risk, for instance during transduction of haematopoietic stem cells. Thus, FV-based SIN vectors require additional genetic modifications in order to avoid RCRs.

Gene Therapy (2004) 11, 465-473. doi:10.1038/sj.gt.3302185

Keywords: foamy virus; self-inactivating vector; retrovirus; replication-competent revertant; bio-safety

\section{Introduction}

Several viruses have intrinsic features predisposing them as efficient vectors for the specific transduction of therapeutic genes into patients or to express vaccine antigens for preventive and/or therapeutic vaccination. ${ }^{1,2}$ However, these advantages are in part counterbalanced by their potential for inducing disease or interfering with essential cellular functions, resulting in serious therapeutic side effects or safety concerns precluding their application in human or veterinary medicine. ${ }^{3}$ In general, several genetic modifications are introduced into the vector genomes, or cis- and transacting elements are split in order to attenuate or abolish replication competence of the vectors. ${ }^{4-6}$ For retroviruses, partial or complete deletion of the U3 promoter of the $3^{\prime}$ long terminal repeat (LTR) is one way to directly abrogate infectivity resulting in self-inactivating (SIN) retroviral vectors; ${ }^{7}$ however, recombination events may lead to replication-competent revertants (RCRs). ${ }^{8-11}$ Such RCRs can be expected to pose, besides the intrinsic insertional mutagenesis potential of retroviral vectors, added risk of altering the expression of cellular genes. Vectors based on apathogenic viruses already possess a substantial degree of biological safety, but under rare

Correspondence: M Löchelt, Abt. Genomveränderung und Carcinogenese, Forschungsschwerpunkt Infektion und Krebs, Deutsches Krebsforschungszentrum, Im Neuenheimer Feld 242, 69120 Heidelberg, Germany Received 09 July 2003; accepted 16 September 2003 conditions genetic manipulation and insertion of therapeutically relevant genes into apparently innocuous viruses can still result in disease potential. ${ }^{12}$

Foamy retroviruses (FVs) are considered apathogenic in their natural host and even after zoonotic transmission into humans. ${ }^{13}$ Due to this intrinsic and well-documented apathogenicity and distinct features of their replication strategy, FVs have a high potential as novel vectors. ${ }^{14-18}$ In addition, disease or oncogenesis due to insertional mutagenesis of FVs has never been described. Recently, we demonstrated applicability, efficiency, and safety of replication-competent feline FV (FFV) vectors for the transfer of vaccine antigens into cats. ${ }^{19}$ More important for medical application, replication-deficient FV-based vectors carrying substantial deletions in structural and regulatory genes have been shown to be especially suited for the long-term transduction of human haematopoietic stem cells. ${ }^{20-22}$ However, the proposed transduction of haematopoietic stem cells or other long-lived cells demands that RCRs are not generated in the patient. ${ }^{23}$ FVs carry a bi-partite packaging sequence extending into gag and pol. ${ }^{24-26}$ Therefore, it is impossible to eliminate all structural gene sequences from FV vectors to avoid recombination between the vector genome and packaging constructs in order to increase bio-safety. Thus, as an alternative or additional approach to destroy replication competence of retroviral vectors, a SIN deletion can be introduced into the $3^{\prime}$ LTR U3 promoter of FV vectors to inhibit structural gene expression. To determine the 
applicability and safety of the SIN strategy in a sensitive detection system, we studied FFV SIN vectors retaining all structural genes. Such studies are mandatory since SIN vectors derived from other retroviruses are already the subject of (pre)clinical trials (summarized in Thomas et $a l^{23}$ ).

To test rigorously the concept of FV-based SIN vectors, we generated FFV SIN vectors still containing all structural and regulatory genes; thus the uptake of a functional promoter by a single recombination event might restore at least part of their replication competence. The applicability and genetic stability of these FFV SIN vectors was studied under cell culture conditions. In the parental replication-competent and in the replicationdeficient SIN vectors (Figure 1a), the internal FFV promoter directs expression of the essential Bel1 transactivator and the Bet protein to which the green fluorescent protein $(g f p)$ marker gene is linked. Thus, expressions of Bel1 and Bet by the empty vectors or Bel1 and the Bet-Gfp fusion protein by Gfp vectors are indicative of vector transduction. ${ }^{27}$

The SIN deletion in the U3 region of the $3^{\prime}$ LTR in the vectors began downstream of bel2/bet and ended 18 residues upstream of the $R$ region (Figure 1a). This strategy maintained the essential bet gene and U3flanking sequences known to be required for reverse transcription and integration, whereas the majority of the U3 promoter together with the TATA box was removed. ${ }^{28-30}$ This SIN deletion was introduced into the infectious FFV genome pCF-7 $\triangle \mathrm{U} 3$ expressed from the heterologous cytomegalovirus (CMV) immediate early (IE) promoter and the Bet-Gfp expression vector pCFBet-Gfp, ${ }^{27}$ both containing a truncated but functionally active U3 promoter lacking residues -725 to -308 relative to the transcriptional start site (Figure 1a). This deletion is compatible with vector replication in vitro and in cats. ${ }^{19,27}$ Each of two independent clones of the novel SIN vectors pCF-SIN (\#2 and \#8) and pCF-Bet-Gfp-SIN (\#6 and \#8) was characterized in detail.

We first determined the titres of the FFV SIN vectors using FAB cells. ${ }^{31}$ FAB cell titration depends on the transduction of the FFV Bel1 transactivator, which is retained in all vectors used. As a consequence, vectortransduced FAB cells score positive. For this purpose, the SIN vector genomes, together with the corresponding replication-competent vectors, were transfected into 293T cells and the titres of cell-free supernatants were determined after 2 days. Data from a representative titration are given in Figure 1b. According to the Bel1mediated activation of $\beta$-galactosidase expression in FAB cells, the titres of the replication-competent vectors $\mathrm{pCF}$ 7 , pCF-7 $\Delta U 3$, and pCF-Bet-Gfp were only slightly higher than those of the corresponding SIN vectors. Additionally, marker gene transfer of the Gfp-transducing vectors was quantified using indirect immunofluorescence (IIF). Titres of Gfp transduction determined by IIF corresponded to those obtained by FAB titration, whereas detection of Gfp by autofluorescence yielded lower values, possibly related to a detection limit of this technique. In summary, the novel FFV-SIN vectors efficiently transduced cells and transferred a reporter gene in cell culture.

Next, we analysed whether RCRs were generated from the promoter-deleted SIN constructs upon extended cultivation of transduced cells. To this end, vector stocks from transfected 293T cells were used to transduce permissive CRFK cells. The transduced cells were serially passaged twice a week and the supernatants were analysed for RCRs by infection of FAB cells.

The results of representative experiments using the empty SIN vectors are presented in Figure 2a. Since the vector inoculum was not removed and since FVs are stable in cell culture medium, ${ }^{32}$ low amounts of input vector were still detectable in the first and second passages in some of the experiments described here. After the 5th (\#8) and 10th (\#2) passages of the SIN vector-transduced cells, FFV infectivities greater than $10^{2} \mathrm{FFU} / \mathrm{ml}$ were clearly detectable (Figure 2a). With a slight temporal delay, syncytia became detectable in the transduced cultures supporting the view that RCRs were formed although the basal concept of the SIN vector construction should prevent RCR development. In other kinetic experiments, RCRs were already detectable after four passages (data not shown).

For Gfp-transducing vectors (Figure 3a), RCRs were clearly detectable at passages 4 (\#8) and 6 (\#6) again paralleled by the subsequent development of syncytia. Whereas two similar experiments revealed comparable kinetics of RCR formation, in a further experiment, no infectious particles were generated even after 10 passages (data not shown). In parallel, we determined the duration of Gfp expression in pCF-Bet-Gfp-SIN-transduced CRFK cell cultures. Over $50 \%$ of the transduced cells were initially positive for Gfp autofluorescence. However, this value based on the less sensitive autofluorescence test decreased to low numbers upon passaging the cells (Figure 3a). We did not analyse whether this decrease was due to silencing of the Gfp expression cassette or a subtle selection against transduced cells. These data clearly demonstrate long-term transduction of a significant number of cells, but RCRs appear fairly consistently from the model SIN vectors after a limited number of cell passages.

In order to characterize generation of RCRs in the long-term transduced CRFK cells, vector-encoded gene expression was analysed with time. CRFK cells transduced with the empty vector pCF-SIN \#2 showed an initial (passages 1-4) strong Bet expression that decreased with time (Figure $2 b$ ). Upon further passages and in parallel with the appearance of RCRs at passage 10, Bet expression increased. Beginning at passage 9 and concomitant to the detection of RCRs, FFV structural Gag and Pol proteins were clearly detectable (data not shown).

For the Gfp-transducing vector pCF-Bet-Gfp-SIN \#6, the pattern of Bet-Gfp expression determined with Gfpspecific antibodies paralleled the situation described above. Initially, Bet-Gfp expression was high allowing even detection of the proteolytically released Gfp protein (Figure 3b). The subsequent decline in Bet-Gfp was followed by a rebounded Bet-Gfp expression at passages 9 and 10, possibly enhanced by RCR-mediated Bel1 transactivator expression and syncytia formation. Immunoblotting with the Bet-specific serum confirmed early strong Bet-Gfp expression (Figure 3c); later, BetGfp was only detectable after overexposure of the blot (not shown). At late passages, the majority of the RCRs no longer expressed the authentic Bet-Gfp fusion protein but only the truncated Bet* protein that had been previously characterized for the parental 
pCF-7AU3

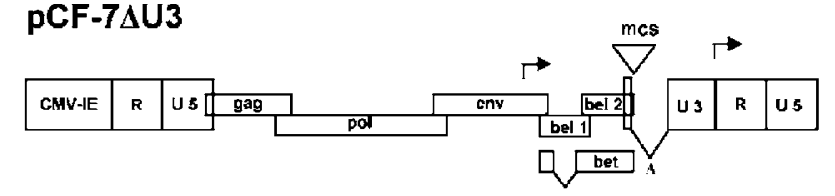

\section{pCF-SIN}

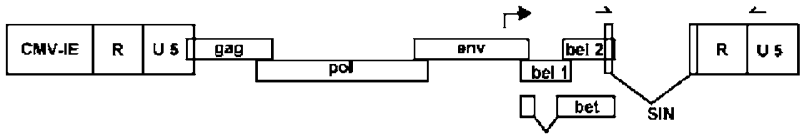

\section{pCF-Bet-Gfp}

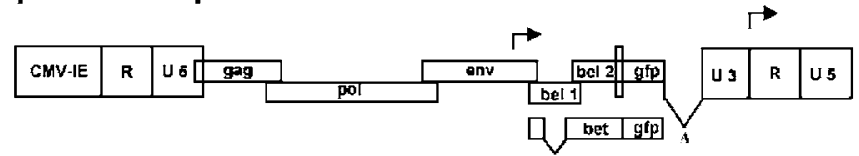

pCF-Bet-Gfp-SIN

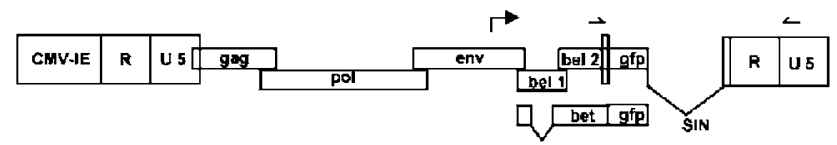

b

Determination of FFV-based vector titres using FAB titration cells

FFV vector genomes

vector titres determined on $\mathrm{FAB}$ cells

used

\begin{tabular}{cc}
\hline pCF-7 & $5 * 10^{7}$ \\
pCF- $\Delta$ U3 & $5 * 10^{6}$ \\
pCF-SIN \#2 & $4 * 10^{6}$ \\
pCF-SIN \#8 & $7 * 10^{5}$ \\
pCF-Bet-Gfp & $2 * 10^{6}$ \\
pCF-Bet-Gfp-SIN \#6 & $2 * 10^{6}$ \\
pCF-Bet-Gfp-SIN \#8 & $2 * 10^{5}$
\end{tabular}

Figure 1 Schematic presentation and analysis of the different FFV vectors used. (a) In the top and middle lanes, the authentic replication-competent and $C M V$-IE promoter-driven FFV vector $p C F-7$ and the derivatives $p C F-7 \triangle U 3$ and $p C F-B e t-G f p$ are shown. ${ }^{27}$ FFV genes, the gfp marker gene, and the LTRs subdivided into the U5, $R$, and U3 regions, are marked by open boxes. The partial U3 deletion in the $3^{\prime}$ LTR compatible with vector replication in vectors $p C F-7 \Delta U 3$ and $p C F-B e t-G f p$ is marked by a broken line (4). The FFV LTR and internal promoters are marked by rectangular arrows. In order to inactivate the U3 promoter, ${ }^{28}$ FFV $3^{\prime}$ LTR sequences from -18 relative to the transcriptional start site (primer SINs; 5'-ATAGCATGCATTATGGTAGATTGTA-

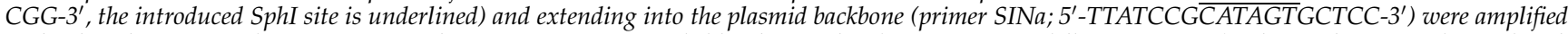
with Pfu polymerase and pCF-7 $4 U 3$ template DNA as recommended by the supplier (Stratagene, Heidelberg, Germany). The amplicon was digested with SphI and ClaI (in the plasmid backbone) and inserted into the correspondingly digested parental vectors $p C F-7 \Delta U 3$ and $p C F-B e t-G f p$. In the resulting clones (bottom line), U3 sequences of the original FFV LTR from -725 to -18 relative to the transcriptional start site including the TATA box were deleted. The resulting clones $p C F-S I N \# 2$ and \#8 and Gfp-transducing vectors $p C F-B e t-G f p-S I N \# 6$ and \#8 were identified and characterized by restriction mapping. The SIN deletion is schematically marked by a broken line. Small horizontal arrows indicate the positions and orientation of PCR primers used to characterize the RCR genomes. (b) In all, $6 \mu \mathrm{g}$ each of the vector genomes depicted above was transfected into 293T cells by calcium phosphate precipitation and the viral/vector titres of the cleared cell culture supernatants were determined 2 days after transfection using FAB cells as described. ${ }^{27}$ Titres are expressed as the number of blue cells per ml cell culture supernatant. ${ }^{31}$ 


\begin{tabular}{|c|c|c|c|c|c|c|c|c|c|c|c|c|c|}
\hline \multirow[b]{2}{*}{ Vector } & \multirow{2}{*}{\multicolumn{2}{|c|}{1}} & \multicolumn{11}{|c|}{ number of passages of transduced CRFK cells } \\
\hline & & & 2 & 3 & 4 & 5 & 6 & 7 & 8 & 9 & 10 & 11 & 12 \\
\hline \multirow{2}{*}{$\begin{array}{c}\text { pCF-SIN } \\
\# 2\end{array}$} & FAB titres & $1.0 * 10^{3}$ & 1 & 0 & 0 & 0 & 0 & 0 & 0 & 2 & $2.0 * 10^{2}$ & $7.0 * 10^{4}$ & n.d. \\
\hline & $\begin{array}{c}\text { syncytia } \\
\text { development }\end{array}$ & - & - & - & - & - & - & - & - & - & - & + & ++ \\
\hline \multirow{2}{*}{$\begin{array}{c}\text { pCF-SIN } \\
\# 8\end{array}$} & FAB titres & $1.0^{*} 10^{3}$ & 0 & 0 & 1 & $1.0^{*} 10^{2}$ & $6.0 * 10^{2}$ & $1.3 * 10^{4}$ & $2.0 * 10^{2}$ & & & & \\
\hline & $\begin{array}{l}\text { syncytia } \\
\text { development }\end{array}$ & - & - & - & - & $(+)$ & $(+)$ & ++ & ++ & & & & \\
\hline
\end{tabular}

b

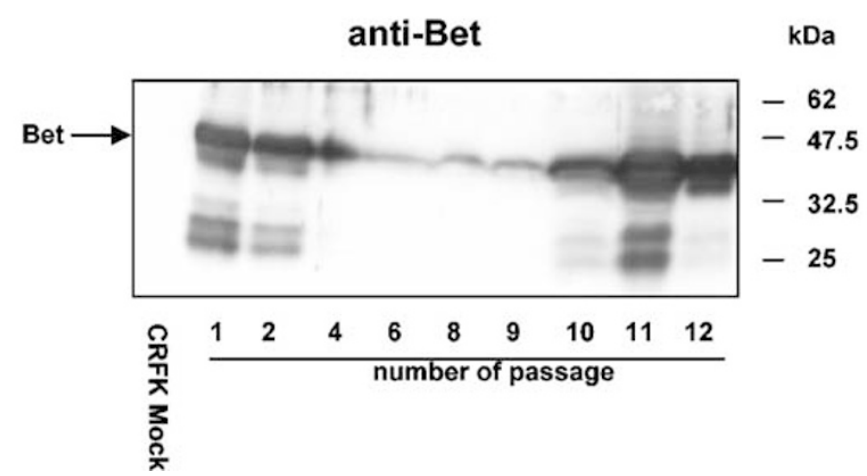

Figure 2 Gene expression and generation of RCRs in CRFK cells transduced with empty pCF-SIN vectors. Vector genomes pCF-SIN \#2 and \#8 were transfected into 293T cells. At 2 days after transfection, $1 \mathrm{ml}$ of the cleared cell culture supernatants was used to transduce subconfluent CRFK cells grown in $10 \mathrm{~cm}$ dishes as described. ${ }^{27}$ The transduced CRFK cell cultures were passaged 1:10 twice a week. For each passage, cleared cell culture supernatants were titrated on FAB cells to detect FFV-specific infectivity resulting from RCRs. In parallel, transduced cells were lysed in $1 \%$ SDS and used for immunoblotting (and PCR analysis; see Figure 4). (a) The titres of RCRs from vectors $p C F-S I N \# 2$ and \#8 as determined using FAB cells are shown. The cultures were terminated at passages 12 (no further titration done, n.d.) and 8 due to strong cytopathic effects (syncytia development). The presence of FFVspecific syncytia is indicated as follows: -: no syncytia; $(+)$ : small cell fusions not indicative of efficient FFV gene expression; + : single, isolated FFVspecific syncytia; + + : strong FFV-specific syncytia formation and cytopathic effects. (b) Regular aliquots of pCF-SIN \#2-transduced CRFK cells taken during the kinetic study (passage numbers are shown below the blot) were subjected to immunoblotting using an FFV Bet antiserum. ${ }^{27,37}$ Bet-specific antibodies were detected by enhanced chemoluminescence (ECl, Amersham Biosciences, Freiburg, Germany). Extracts from mock-and FFV-infected (data not shown) CRFK cells served as controls. Molecular masses of marker proteins (broad range prestained protein marker, New England Biolabs, Frankfurt, Germany) separated in parallel are given at the right-hand margin; the arrow indicates the position of FFV Bet. To allow detection of Bet expression between passages 4 and 9, the blot was intentionally overexposed. Loading of corresponding protein amounts was confirmed in all experiments by Coomassie staining (data not shown).

replication-competent vector pCF-Bet-Gfp. ${ }^{27}$ Bet $^{*}$ is generated when $g f p$ sequences are deleted from the vector, and consists of the complete bet sequence plus residues derived either from gfp or the vector. ${ }^{27}$

RCR-producing cells transduced with vector pCF-BetGfp-SIN \#8 (compare RCR titres, Figure 3a) expressed at the fifth passage not only Bet* (Figure $3 \mathrm{~d}$ ) but also the FFV structural p52 and p48 Gag proteins, the polencoded p70 PR-RT-RNaseH fusion protein, and the uncleaved Pol precursor p127 ${ }^{\mathrm{Pol}}$ (Figure 3e). The data show that RCR-generating cultures expressed FFV vector proteins and that a fraction of cells retained expression of the authentic Bet-Gfp fusion protein. The presence of intact $g f p$ sequences in late passage cultures was confirmed by PCR analysis using $g f p$-specific primers (data not shown).

To define the molecular events that have led to the generation of the RCRs, DNA taken at every passage of the transduced CRFK cells was analysed by PCR. This PCR assay amplified a region from the $3^{\prime}$ end of bet and extending into the intact U5 region encompassing the area where the SIN deletion was introduced (see Figure 1).

Initially, RCR proviral genomes from pCF-SIN \#2transduced cells (compare Figure 2) were analysed. The authentic vector genome was clearly detectable by PCR and gel analysis until passage 9, whereas additional PCR products (amplicons) of larger sizes appeared (Figure $4 a$ ). One of these amplicons was of cellular origin as determined by DNA sequencing. At late passages, a single DNA band of 615 base pairs (bp, white arrow) became the prominent amplification product; it was cloned and sequenced. Sequence analysis revealed (Figure 4b, bottom line) that a 269-bp-long part of the CMV-IE promoter (bold-face residues -269 to -1 relative to the CMV-IE transcriptional start site) had replaced the SIN deletion. This sequence most probably originated from the CMV-IE promoter directing vector genome expression in the original plasmid clone, as schematically indicated in Figure $4 \mathrm{~b}$. During reverse transcription of the vector genome, this novel chimeric LTR promoter had replaced the original SIN deletion, resulting in the RCR described. Remarkably, clear sequence homology between the $5^{\prime}$ and $3^{\prime}$ ends of the inserted CMV-IE promoter sequence and the target site in the SIN-deleted $3^{\prime}$ LTR (marked by dots below the sequence) might have guided recombination. According to the PCR data, this genome had replaced all other genotypes during passages. Since the vector titres at these passages were significantly lower than those of FFV vectors with the 


\begin{tabular}{|c|c|c|c|c|c|c|c|c|c|c|}
\hline \multirow[b]{2}{*}{ Vector } & \multirow{2}{*}{\multicolumn{2}{|c|}{1}} & \multicolumn{8}{|c|}{ number of passages of transduced CRFK cells } \\
\hline & & & 2 & 3 & 4 & 5 & 6 & 7 & 8 & 9 \\
\hline \multirow{3}{*}{$\begin{array}{l}\text { pCF-Bet- } \\
\text { Gfp-SIN \#6 }\end{array}$} & FAB titres & $1.0 * 10^{3}$ & $1.0 * 10^{\prime}$ & 0 & $1.0^{*} 10^{1}$ & $2.0^{*} 10^{\circ}$ & $1.0^{*} 10^{2}$ & $2.0 * 10^{2}$ & $1.0 * 10^{3}$ & $2.0 * 10^{4}$ \\
\hline & $\begin{array}{c}\text { syncytia } \\
\text { development }\end{array}$ & $\cdot$ & - & - & - & - & - & - & ++ & ++ \\
\hline & $\begin{array}{c}\text { Gfp-positive } \\
\text { cells }\end{array}$ & $>50 \%$ & $50 \%$ & $10-20 \%$ & $1-5 \%$ & $1-5 \%$ & $1 \%$ & $1-5 \%$ & n.d. & $1-5 \%$ \\
\hline \multirow{3}{*}{$\begin{array}{l}\text { pCF-Bet- } \\
\text { Gfp-SIN \#8 }\end{array}$} & FAB titres & $1.0 * 10^{3}$ & $1.0 * 10^{1}$ & $1.0 * 10^{1}$ & $1.0 \cdot 10^{3}$ & $3.0 .10^{3}$ & $1.0 * 10^{5}$ & & & \\
\hline & $\begin{array}{c}\text { syncytia } \\
\text { development }\end{array}$ & - & - & - & + & ++ & ++ & & & \\
\hline & $\begin{array}{l}\text { Gfp-positive } \\
\text { cells }\end{array}$ & $>50 \%$ & $50 \%$ & $10-20 \%$ & $1-5 \%$ & $1-5 \%$ & $1 \%$ & & & \\
\hline
\end{tabular}

pCF-Bet-Gfp-SIN \#6

b

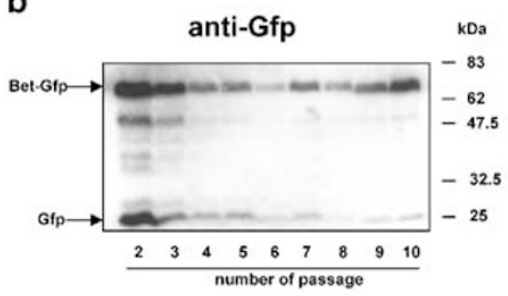

c

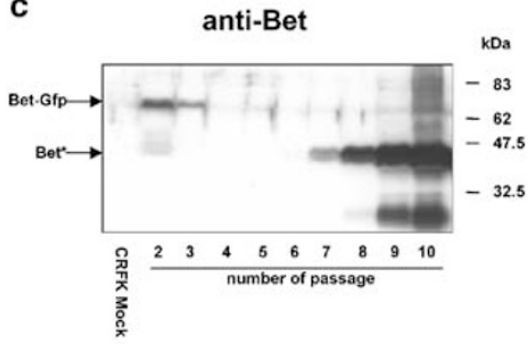

pCF-Bet-Gfp-SIN \#8

d

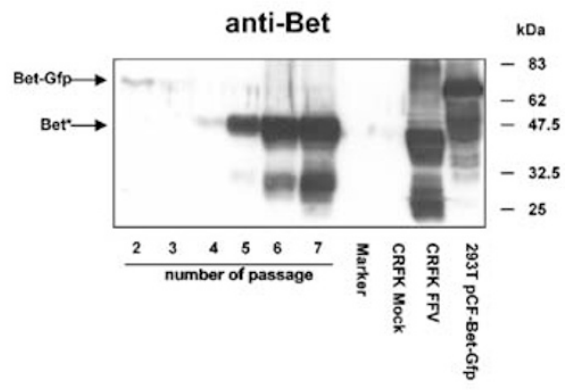

e

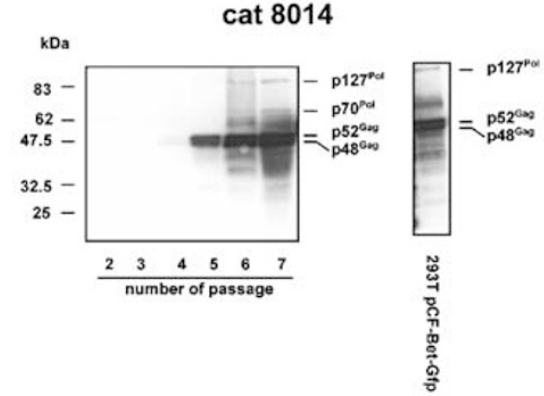

Figure 3 Gene expression and generation of RCRs in CRFK cells transduced with Gfp expression vectors $p C F-B e t-G f p-S I N$. Vector genomes $p C F-B e t-G f p-$ SIN \#6 and \#8 were transfected into $293 \mathrm{~T}$ cells. At 2 days after transfection, $1 \mathrm{ml}$ of the cleared cell culture supernatants was used to transduce subconfluent CRFK cells as described above. Transduced CRFK cells were passaged 1:10 twice a week, and cleared cell culture supernatants were titrated on FAB cells to detect RCRs. Transduced cells were lysed in 1\% SDS and used for immunoblotting (and PCR analysis; see Figure 5). (a) The titres of RCRs from vectors $p C F-B e t-G f p-S I N \# 6$ and \#8 as determined using FAB cells are shown. The cultures were terminated at passages 9 and 6 due to strong cytopathic effects. The appearance of FFV-specific syncytia is indicated as follows: $-:$ no syncytia; $+:$ single, isolated syncytia; ++ : strong syncytia formation and cytopathic effects. Marker gene expression was determined by Gfp autofluorescence of transduced cells, and the fraction of cells positive for Gfp autofluorescence is indicated in percent. Regular aliquots of pCF-Bet-Gfp-SIN \#6- $(b, c)$ and \#8-transduced CRFK cells $(d, e)$ taken during the kinetic study (passage numbers are shown below the blots) were subjected to immunoblotting. The following antisera were used: (b) anti Gfp; (c, d) anti-FFV Bet; (e) cat serum against FFV Gag and Pol proteins (designated cat 8014). ${ }^{37,38}$ Extracts from mock-and FFV-infected CRFK cells and pCF-Bet-Gfp-transfected $293 T$ cells served as controls. Bound antibodies were detected by ECl. Molecular masses of marker proteins separated in parallel are given. The positions of the Bet-Gfp fusion protein, the proteolytically released Gfp protein, the rearranged Bet* protein, and the FFV $p 48^{\mathrm{Gag}}$, $p 52^{\mathrm{Gag}}$, $p 70^{\mathrm{Pol}}$, and $p 127^{\mathrm{Pol}}$ precursor proteins are marked at the opposite margin. Some blots were intentionally overexposed to allow detection of low-level vector gene expression before the appearance of RCRs.

intact or truncated FFV U3 promoters, the 269-bp-short CMV-IE promoter fragment allowed a suboptimal, strongly attenuated replication. A similar conclusion has been recently drawn from CMV-IE promoter-driven HFV mutants. ${ }^{33}$

Next, we analysed by PCR the kinetics of RCR genomes from Gfp-transducing vectors. Data from the kinetics described in Figure 3 for vectors pCF-Bet-GfpSIN \#6 and \#8 are shown in Figure 5a. Similar to the situation with the SIN vector backbones, several different amplification products corresponding to diverse genotypes appeared during serial passages. Importantly, different amplicons were generated in repeated experiments with the same or different subclones (data not shown; see Figure 5a). The original genome was reproducibly replaced by rearranged larger or smaller RCRs, or the original sequence was competed out during PCR amplification by the RCR genomes. In the culture 


\section{pCF-SIN \#2}

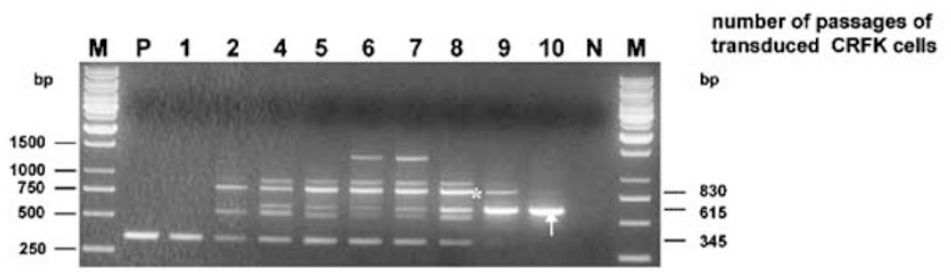

b

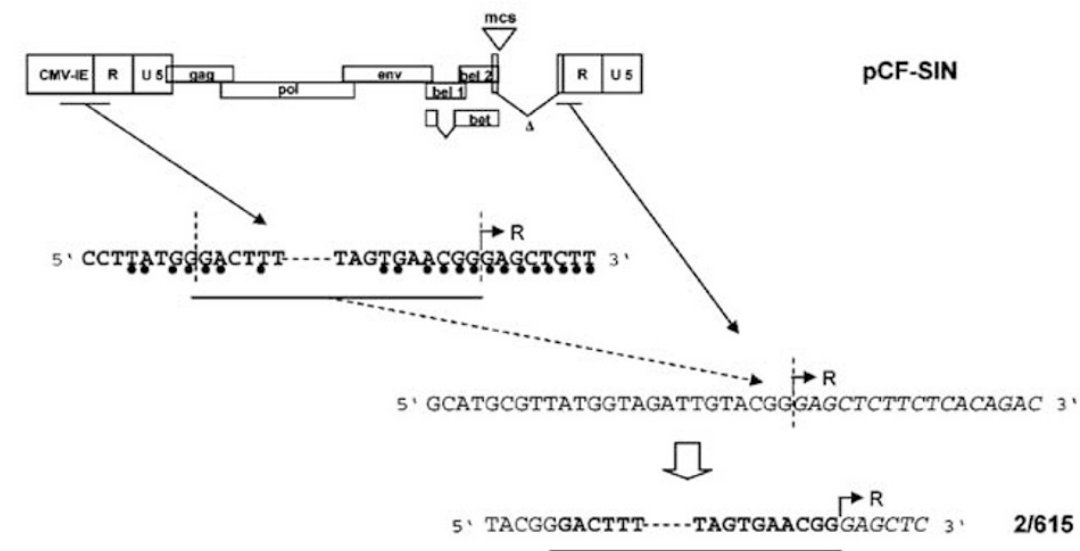

Figure 4 Detection and analysis of RCR genomes from CRFK cells transduced with the empty $p$ CF-SIN \#2 vector. Parts of the SDS lysates of $p C F-S I N \# 2-$ transduced CRFK cells (see Figure 2) were used for PCR amplification using primers FeFV10583s (5'-CAAAAGTGATACTTCCTG-3') and U5/11578a (5'-GAGTTCTGGCTTCAGAC-3'; see Figure 1a for primer localization). PCR conditions using Taq polymerase were as recommended by the supplier (Stratagene, Heidelberg, Germany): $100 \mathrm{ng} D N A, 35$ cycles of $94^{\circ} \mathrm{C}$ for $30 \mathrm{~s}, 48^{\circ} \mathrm{C}$ for $40 \mathrm{~s}, 75^{\circ} \mathrm{C}$ for $3 \mathrm{~min}$. (a) Identical aliquots of the PCR reactions were subjected to agarose gel electrophoresis and amplicons were visualized by ethidium bromide staining. The passage numbers are shown above the gel. In lane $P$, a plasmid control of the $p C F-S I N \# 2$ vector was amplified and lane $N$ did not contain any template DNA. In lane $M$, molecular size markers (1 kb gene ruler, MBI Fermentas, St Leon-Rot, Germany) were separated, and the bands of 250, 500, 750, 1000, and 1500 bp are marked at the left-hand margin. The bands labelled with an asterisk (830 bp) and an arrow (615 bp) were isolated (Gel extraction kit, Qiagen, Hilden, Germany), subcloned into $p$ CR2.1TOPO (Invitrogen, Groningen, The Netherlands), and sequenced using vector-derived flanking primers. The 345-bp-long amplicon representing the original $p C F-$ SIN sequence gradually decreased upon repeated passages. (b) The 830-bp-long band (asterisk) turned out to represent unspecifically co-amplified cellular $D N A$. The unique band of $615 \mathrm{bp}$ at passage 10 represented the rearranged RCR 2/615 genome schematically shown in the bottom line labelled 2/615. CMV-IE promoter sequences from -269 to -1 relative to the transcriptional start site are in bold-face letters and underlined, and the dashes represent sequences that are not shown. Flanking sequences are derived from the deleted $3^{\prime}$ LTR of the SIN vector. Above, the original sequences of the CMV-IE promoter (bold-face letters) directing expression of the vector genome and the 3' LTR of the SIN vector are shown together with a schematic presentation of the $p C F-S I N$ genome. The stippled and open arrows indicate how the sequences might have recombined. The $R$ sequences are shown in italics, and the broken arrows mark the start sites of transcription.

derived from clone \#6, the predominating amplicons were 437 and $1215 \mathrm{bp}$ in size (arrowheads) whereas those from the vector pCF-Bet-Gfp-SIN \#8 had sizes of 555 and $996 \mathrm{bp}$ (arrows). These major amplicons $(6 / 437,6 / 1215,8 / 555$, and 8/996) were characterized by sequence determination. Amplicons 8/996, 8/555, and amplicon 6/406 (from an independent experiment) were cloned and sequenced; the results are schematically presented in Figure 5b. Amplicons 6/437 and $6 / 1215$ were directly sequenced without prior cloning.

In all clones analysed (Figure 5b), different parts of the CMV-IE promoter substituted the original SIN deletion, conferring replication competence to the RCRs. Consistently, the $3^{\prime}$ terminal site where CMV-IE- and FFV $3^{\prime}$ LTR sequences were fused ( $3^{\prime}$ junction) was identical to that described for the empty SIN clone (Figure 4b). This confirmed that the high local homology between both sequences before recombination and the functional selection for replication-competent genomes favoured this site. In contrast, the $5^{\prime}$ junction was not conserved relative to CMV and vector sequences, except that in all clones the bet gene was left fully intact as already observed in another study. ${ }^{27}$ In all RCR genomes analysed, the majority of the $g f p$ gene was deleted and replaced by different parts of the CMV-IE promoter. In RCR 6/1215, even pAT153 plasmid-derived sequences located upstream of the CMV-IE promoter (indicated in Figure $5 \mathrm{~b}$ ) were taken up alongside to the entire CMV-IE promoter. Since amplicons 6/ 437 and 6/1215 could be directly sequenced, the corresponding bands represent unique RCR clones generated by single recombination events and do not represent a mixed population of heterogeneous DNAs of similar length. Whereas in most RCR clones some sequence homologies were detectable before recombination at the $5^{\prime}$ junction, almost no homology before recombination was detectable for clones $8 / 555$ and $8 / 996$.

It is open whether genomes $8 / 555$ and $6 / 437$ carrying the smaller CMV-IE promoter fragments devoid of the CMV enhancers ${ }^{34}$ contributed to the FFV titres 
a

\section{pCF-Bet-Gfp-SIN \#6}

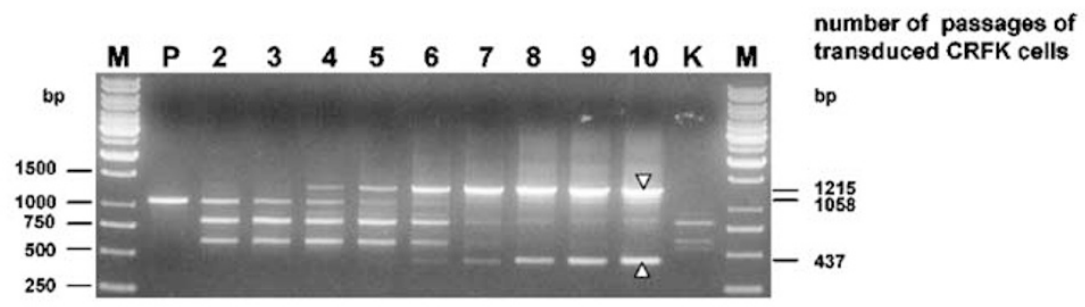

pCF-Bet-Gfp-SIN \#8

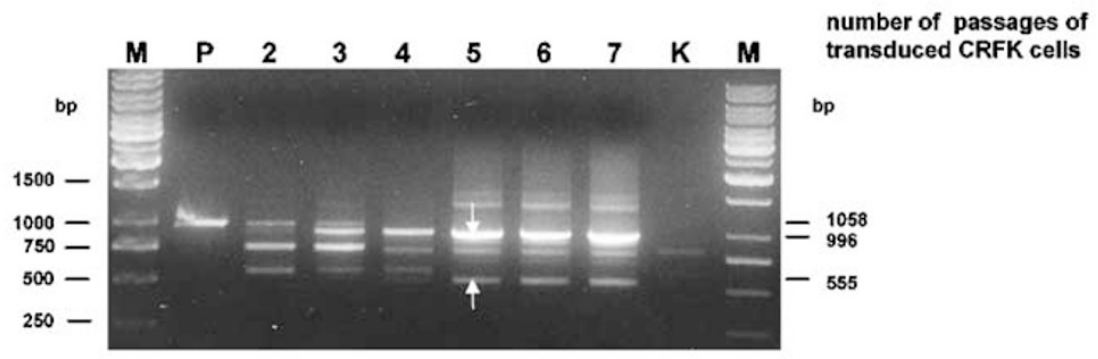

b
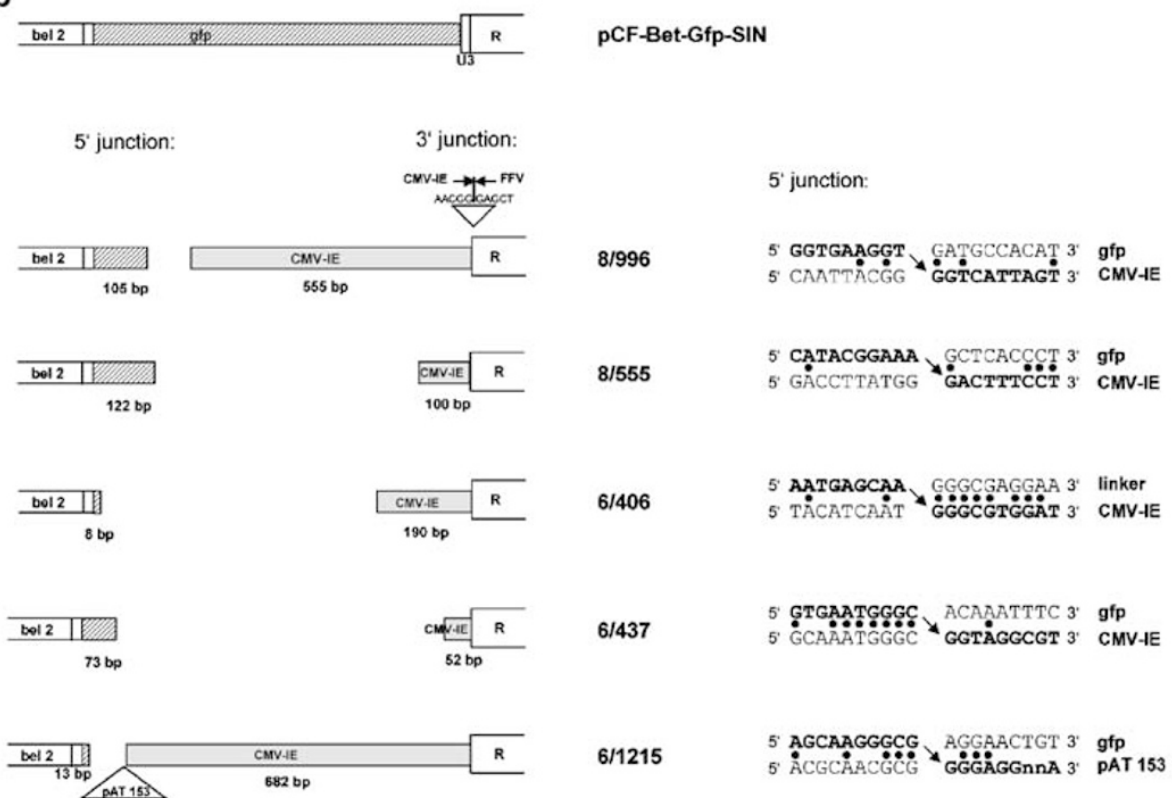

Figure 5 Detection and analysis of RCR genomes from CRFK cells transduced with vectors $p$ CF-Bet-Gfp-SIN \#6 and \#8. Parts of the SDS lysates from pCF-Bet-Gfp-SIN \#6- and \#8-transduced CRFK cells (see Figure 3) were used for PCR with primers FeFV10583s and U5/11578a as described in the legend to Figure 4. (a) Aliquots of the PCR reactions were separated on agarose gels and visualized by ethidium bromide staining. The passage numbers are shown above the agarose gel. In lane P, a plasmid control of the $p C F-B e t-G f p$-SIN \#6 vector was amplified and lane K contained DNA from mock-infected CRFK cells as template. In lane M, molecular size markers were separated, and the bands of 250, 500, 750, 1000, and 1500 bp are marked at the left-hand margin. The $p C F-B e t-G f p-S I N$ \#6-derived bands of 1215 and 437 bp (labelled by arrowheads) were gel-extracted and directly sequenced using the PCR primers. The pCF-Bet-Gfp-SIN \#8-derived bands of 555 and 996 bp (labelled by arrows) were isolated, subcloned, and sequenced as described in Figure 4. In both $p C F-$ Bet-Gfp-SIN clones, the 1058-bp-long amplicon representing the original sequence gradually decreased upon repeated passages. In (b), the data of the sequence analyses are schematically presented. In the top line, the structure of the bet-gfp-SIN region of the original SIN clones is presented. Below, the structures of the RCRs described in (a) and clone 6/406 are shown. In all clones, parts of the gfp sequence (hatched boxes) were retained; the size of the truncated gfp gene is given in bp. Consistently, CMV-IE promoter fragments of different sizes (shaded boxes) were detected. In all clones, the 3' junction was identical to that described in Figure $4 b$ and shown for clone 2/615. The $5^{\prime}$ ends and the sizes of the CMV-IE inserts (as given below the boxes) differed significantly and extended even into pAT153 plasmid sequences in clone 6/1215. The gfp and CMV-IE (and pAT153) sequences that recombined are given for each of the RCRs. The gap represents the site where gfp (top) and CMV-IE or pAT153 sequences were fused (bottom). The co-linear sequence of the RCRs is given in bold-face letters switching at the junction site from the gfp (top) to the CMV-IE (or pAT153) sequence (bottom) as indicated by the arrow. The dots between the sequences mark identical residues that might have guided recombination. 
or whether only the coexisting RCRs 8/996 and 6/1215 displayed a reduced but clearly detectable replication competence. Probably, the smaller CMV-IE promoter fragments simply represent defective genomes present in the transduced cells. RCR clone 6/406 may have a reduced replication competence since some CMV-IE enhancers are retained and since a similar RCR of MLV displayed replication competence. ${ }^{34,35}$

In summary, in the FFV SIN vector-derived RCRs, CMV-IE promoter sequences between -682 to -1 and -52 to -1 were taken up. These genetic changes partially restored LTR promoter activity, thus allowing attenuated RCR replication. In some cases, genetically rearranged genomes were already detectable in freshly transduced cells (data not shown). During further cell passages, one or two dominant genotypes replaced the other RCR genomes. None of the major RCRs formed retained the intact $g f p$ gene. However, the authentic vector genome persisted in the transduced cultures resulting in low but detectable numbers of cells expressing the marker gene as confirmed by a $g f p$-specific PCR (data not shown). These transduced cells persisted over extended periods of time; however, selection against them apparently took place.

The genetic rearrangements between the SIN deletion site and the CMV-IE promoter occurred in the vast majority of cases in regions with distinct, local sequence homology. This is especially true for the $3^{\prime}$ junction that is common in all clones and most likely strongly favoured by the repeated $\mathrm{R}$ and flanking sequences at the $5^{\prime}$ and $3^{\prime}$ ends of the genome. The early appearance of rearranged genomes and the uptake of plasmid sequences into RCR 6/1215 may indicate that recombination had already occurred in the transfected vector genomes. However, recombination between vector RNA genomes is formally not excluded by this observation, ${ }^{36}$ since RNA transcripts extending into plasmid sequences may be generated by inefficient termination of vector mRNA synthesis in the $3^{\prime}$ LTR carrying the SIN deletion.

The findings presented here for FV-based vectors are different from those described for MLV vectors, $, 9,10,35$ since RCRs appeared more rapidly with FFV SIN vectors. This is surprising since the FFV SIN deletion removed promoter sequences from -18 to -725 , whereas those for MLV were much smaller ( -150 to -357$)$ removing only enhancer elements and hardly affecting the core promoter. $^{35}$

We show that RCRs develop rapidly in FV SIN vectors containing all structural genes. Since the recombination events leading to RCRs obviously depended on local sequence homologies, it may be possible to suppress recombination events partially as previously described by reducing homologous regions. ${ }^{11}$ However, recombination also took place at sites with almost no homology, and FV-derived vectors exclusively carrying a SIN inactivation are expected to be intrinsically not safe enough for the transduction of long-lived cells, for instance stem cells. Therefore, FV SIN vectors also deleted in one or more structural genes, a strategy already used for FV-based vectors, ${ }^{20,22}$ should display a degree of biological safety required for application in men and animals; experiments to construct and analyse the corresponding vectors are presently underway.

\section{Acknowledgements}

We thank Jennifer Reed, Jürgen Kleinschmidt, Nadine Kirchner (DKFZ), and Allison German (Bristol University, UK) for critically reading the manuscript, and Harald zur Hausen for continuous support.

\section{References}

1 Kay MA, Glorioso JC, Naldini L. Viral vectors for gene therapy: the art of turning infectious agents into vehicles of therapeutics. Nat Med 2001; 7: 33-40.

2 Schnell MJ. Viral vectors as potential HIV-1 vaccines. FEMS Microbiol Lett 2001; 200: 123-129.

3 Chang L-J. Pest not guest. Gene Therapy 2003; 10: 193-194.

4 Verma IM, Somia N. Gene therapy - promises, problems and prospects. Nature 1997; 389: 239-242.

5 Somia N, Verma IM. Gene therapy: trials and tribulations. Nat Rev Genet 2000; 1: 91-99.

6 Buchschacher GL. Introduction to retroviruses and retroviral vectors. Somat Cell Mol Genet 2001; 26: 1-11.

$7 \mathrm{Yu}$ SF et al. Self-inactivating retroviral vectors designed for transfer of whole genes into mammalian cells. Proc Natl Acad Sci USA 1986; 83: 3194-3198.

8 Olson P, Temin HM, Dornburg R. Unusually high frequency of reconstitution of long terminal repeats in U3-minus retrovirus vectors by DNA recombination or gene conversion. J Virol 1992; 66: 1336-1343.

9 Reuss FU, Berdel B, Heber R, Ploss M. Enhancer-deficient amphotropic murine leukemia virus and recombinants with heterologous transcription elements can be efficiently amplified and detected in Mus dunni fibroblasts. Gene Therapy 2002; 9: $1183-1188$.

10 Reuss FU, Berdel B, Ploss M, Heber R. Replication of enhancerdeficient amphotropic murine leukemia virus in human cells. Proc Natl Acad Sci USA 2001; 98: 10898-10903.

$11 \mathrm{Yu}$ SS et al. Construction of a retroviral vector production system with the minimum possibility of a homologous recombination. Gene Therapy 2003; 10: 706-711.

12 Jackson RJ et al. Expression of mouse interleukin- 4 by a recombinant ectromelia virus suppresses cytolytic lymphocyte responses and overcomes genetic resistance to mousepox. J Virol 2001; 75: 1205-1210.

13 Meiering CD, Linial ML. Historical perspective of foamy virus epidemiology and infection. Clin Microbiol Rev 2001; 14: 165-176.

14 Bieniasz PD et al. Gene transfer using replication-defective human foamy virus vectors. Virology 1997; 235: 65-72.

15 Hill CL, Bieniasz PD, McClure MO. Properties of human foamy virus relevant to its development as a vector for gene therapy. J Gen Virol 1999; 80: 2003-2009.

16 Mergia A, Heinkelein M. Foamy virus vectors. Curr Top Microbiol Immunol 2003; 277: 131-159.

17 Bodem J, Löchelt M, Yang P, Flügel RM. Regulation of gene expression by human foamy virus and potentials of foamy viral vectors. Stem Cells 1997; 15: 141-147.

18 Linial M. Why aren't foamy viruses pathogenic? Trends Microbiol 2000; 8: 284-289.

19 Schwantes A et al. Application of chimeric feline foamy virusbased retroviral vectors for the induction of antiviral immunity in cats. J Virol 2003; 44: 7830-7842.

20 Vassilopoulos G, Trobridge G, Josephson NC, Russell DW. Gene transfer into murine hematopoietic stem cells with helper-free foamy virus vectors. Blood 2001; 98: 604-609.

21 Trobridge G, Vassilopoulos G, Josephson N, Russell DW. Gene transfer with foamy virus vectors. Methods Enzymol 2002; 346: 628-648. 
22 Leurs $\mathrm{C}$ et al. Comparison of three retroviral vector systems for transduction of nonobese diabetic/severe combined immunodeficiency mice repopulating human CD34(+) cord blood cells. Hum Gene Ther 2003; 14: 509-519.

23 Thomas CE, Ehrhardt A, Kay MA. Progress and problems with the use of viral vectors for gene therapy. Nat Rev Genet 2003; 4: 346-358.

24 Trobridge $\mathrm{G}$ et al. Improved foamy virus vectors with minimal viral sequences. Mol Ther 2002; 6: 321-328.

25 Erlwein O, Bieniasz PD, McClure MO. Sequences in pol are required for transfer of human foamy virus-based vectors. J Virol 1998; 72: 5510-5516.

26 Heinkelein $\mathrm{M}$ et al. Improved primate foamy virus vectors and packaging constructs. J Virol 2002; 76: 3774-3783.

27 Schwantes A, Ortlepp I, Löchelt M. Construction and functional characterization of feline foamy virus-based retroviral vectors. Virology 2002; 301: 53-63.

28 Bodem J, Löchelt $M$, Delius H, Flügel RM. Detection of subgenomic cDNAs and mapping of feline foamy virus mRNAs reveals complex patterns of transcription. Virology 1998; 244: 417-426.

29 Löchelt M. Foamy virus transactivation and gene expression. Curr Top Microbiol Immunol 2003; 277: 27-61.

30 Rethwilm A. The replication strategy of foamy viruses. Curr Top Microbiol Immunol 2003; 277: 1-26.

31 Zemba $\mathrm{M}$ et al. Construction of infectious feline foamy virus genomes: cat antisera do not cross-neutralize feline foamy virus chimera with serotype-specific Env sequences. Virology 2000; 266: 150-156.

32 Li Z, Yang P, Liu H, Li WX. A new indicator cell line to monitor human foamy virus infection and stability in vitro. Intervirology 2002; 45: 79-84.

33 Schenk T, Enssle J, Fischer N, Rethwilm A. Replication of a foamy virus mutant with a constitutively active U3 promoter and deleted accessory genes. J Gen Virol 1999; 80: 1591-1598.

34 Boshart $\mathrm{M}$ et al. A very strong enhancer is located upstream of an immediate early gene of human cytomegalovirus. Cell 1985; 41: 521-530.

35 Reuss FU, Heber R, Ploss A, Berdel B. Amphotropic murine leukemia virus replication in human mammary epithelial cells and the formation of cytomegalovirus-promoter recombinants. Virology 2001; 291: 91-100.

36 Chong H, Starkey W, Vile RG. A replication-competent retrovirus arising from a split-function packaging cell line was generated by recombination events between the vector, one of the packaging constructs, and endogenous retroviral sequences. J Virol 1998; 72: 2663-2670.

37 Alke A et al. The bet gene of feline foamy virus is required for virus replication. Virology 2001; 287: 310-320.

38 Alke A et al. Characterization of the humoral immune response and virus replication in cats experimentally infected with feline foamy virus. Virology 2000; 275: 170-176. 